\title{
Outer Synchronization of Drive-Response Complex-Valued Complex Networks via Intermittent Pinning Control
}

\author{
Xuefei Wu $\mathbb{D},{ }^{1}$ Jianwen Feng $\mathbb{D},^{2}$ and Zhe Nie $\mathbb{C D}^{3}$ \\ ${ }^{1}$ Artificial Intelligence School, Shenzhen Polytechnic, Shenzhen 518055, China \\ ${ }^{2}$ College of Mathematics and Statistics, Shenzhen University, Shenzhen 518060, China \\ ${ }^{3}$ Digital Creativity and Animation School, Shenzhen Polytechnic, Shenzhen 518055, China \\ Correspondence should be addressed to Zhe Nie; niezhe@szpt.edu.cn
}

Received 8 October 2020; Revised 24 December 2020; Accepted 27 January 2021; Published 8 February 2021

Academic Editor: Guang Li

Copyright (C) 2021 Xuefei Wu et al. This is an open access article distributed under the Creative Commons Attribution License, which permits unrestricted use, distribution, and reproduction in any medium, provided the original work is properly cited.

This paper is concerned with the outer exponential synchronization of the drive-response complex dynamical networks subject to time-varying delays. The dynamics of nodes is complex valued, the interactions among of the nodes are directed, and the two coupling matrices in the drive system and the response system are also different. The intermittent pinning control is proposed to achieve outer exponential synchronization in the aperiodical way. Some novel sufficient conditions are derived to guarantee outer exponential synchronization of the considered complex-valued complex networks by using the Lyapunov functional method. Finally, two numerical examples are presented to illustrate the effectiveness of the proposed control protocols.

\section{Introduction}

Under the background of surging development of network technique and information technique, a lot of attention has been drawn on the research of complex networks by more and more scholars. Modeling for the complex networks $[1-3]$ is an essential part of deploying real network in our life. At the same time, as an important collective attribute of a network, synchronization is widely used which includes robot synchronous operation, sensor clock synchronization, and power system frequency synchronization. The research of the synchronization type in this area has also been the concern of many scholars, and many great results have been achieved in recent years such as synchronization, outer synchronization, almost sure synchronization, projection synchronization, and quasisynchronization [4-11].

Generally, we consider the network synchronization in two ways. First is the synchronization generating inside a network which is focused on the unity of all the nodes. We call it "inner synchronization." The exiting research studies mostly focus on synchronization, partial synchronization, and so on. Second is synchronization achieving between two or more complex networks in the drive-response way, no matter whether the inner synchronization of one same network is realized or not. This type of synchronization, as the name implies, is called "outer synchronization." It is easy enough to think of examples in real life. If we regard the developed countries and developing countries as two networks, one can see that the two networks will eventually achieve synchronization with the exchange visits between the countries frequently. In the same way, we take the couple as two different networks and take their appearance, personality, values, and outlook on life as different nodes, through long running-in, and two networks will also be synchronized. Recently, some studies about outer synchronization of complex networks have sprung up. For example, Ray and Roychowdhury [12] investigated a class of outer synchronization between two different networks with different nodes. Based on this paper, Lu et al. [13] present an analytical method of outer synchronization of local coupled dynamical networks by using a pinning impulsive controller and regrouping method. Furthermore, outer synchronization between two uncertain networks with different node numbers, using the method of adaptive scaling function, is further investigated [14]. Zhang et al. [15] study the generalized outer synchronization of coupled complex 
networks, considering nondissipative and different timevarying coupling delays. These results provide many valuable references for us studying the outer synchronization. It is important to note that the most of the existing research focuses on the same network structure of outer network synchronization for the drive-response systems.

For a long time, the research of complex network synchronization in real number domain has attracted lots of scholars' attention, but in fact, the studies about the complex-domain deserve more attention for its great potential and vast development foreground. For example, in the field of fuzzy neural network, due to the obvious uncertainty and fuzziness, the complex signal cannot be processed by the real domain, and at this point, the complex-domain must be considered. Zhang et al. [16] discussed the synchronization for a new type of fully complex-valued networks including the linear feedback control in a finite time and coupling delay. In [17], Wu et al. probed using the pinning control strategy to realize the synchronization of complex-variable network. The relative conclusion in this letter involves realdomain and complex-domain networks. Sun and Xu et al. investigated the synchronization of complex-variable networks with fractional order $[18,19]$. Among them, Sun et al. mainly discussed the real combination asymptotical synchronization of three fractional-order complex-variable chaotic systems based on the related theories [18], and Xu et al. used the fractional-order techniques and the correlation control strategy to implement fractional-order complex-domain network synchronization [19].

In the previous relative works, the dynamics of the nodes and the coupling matrices of the networks are identical, and it is more common and easier to discuss the synchronization of two networks, but that is not the case; most of the time, the structures of coupling matrices of drive-response complex dynamical networks are not the same. For example, in the ecological network, the two systems which are constituted by a predator and prey are usually in imbalance, leading to the extinction of a species or ecological environment being changed. Zhou et al. contributed a valuable instance in which they construct two classes of small-world and WS networks through taking some fiber lasers with hyperchaos features as nodes [20]. The authors take the relationship of three people as an example to explain the notion of differentdimensional node clearly [21]. Zhang et al. first investigate the stabilization issues including time-varying and interrupted complex networks with uncertain nonlinearities, in which they design some new stabilization controllers [22]. Then, they research the outer synchronization between two complex networks with different time-varying coupling delays. They design two controllers for their drive system and response system by using some particular technique called "open-plus-closed-loop" [23].

The aperiodic intermittent control is a very good type of control strategy that only constrains the boundaries of the time interval between samples. Compared to the real-domain one, the complex-domain systems have more utility value, and at the same time, the sampling in the aperiodical way is also more universal than in the periodical case. Cai et al. investigated the outer synchronization between two complex dynamical networks by using aperiodically adaptive intermittent pinning control [24]. Lei et al. constructed a new piecewise auxiliary function to realize the outer synchronization of two general complex networks delayed by using an aperiodically adaptive intermittent control scheme [25]. Unfortunately, it has been found that the system given by the authors is inapplicable to the complex-value complex dynamical networks. Compared with existing real-value complex dynamical networks, the complex-value case is more challenging since the state of the dynamics is complex.

Motivated by the previous research, in this manuscript, we investigate the exponential outer-synchronization issue of complex-valued complex dynamical networks, in which the coupling matrices of the drive-response systems are different. The major differences are that the state of the systems is complex-valued, the intermittent control is aperiodic, and the coupling matrices are different in the drive and response system. The main contributions are highlighted as follows: Firstly, a new complex-valued complex dynamical network model with nondelays and time-varying delays coupling is formulated to model the real networks. Secondly, by using the Lyapunov functional method, stability theory, complex-valued differential equations, and other related mathematics theories and techniques, some practical sufficient conditions are relieved to ensure exponential synchronization of our networks. Meanwhile, we have adopted a flexible control strategy to reduce the cost of synchronization. Finally, we give some numerical simulation to verify our results.

The frame structure of this manuscript is as follows. In Section 2, this part is the prophase work, including the introduction and description of the various definitions and theorems, quotes, hypothesis, and so on. In Section 3, combining with the previous theorem, assumptions, and so on, some effective criteria of complex network synchronization are deduced and related inference is given. In Section 4 , we give some numerical simulation to verify our results. At last, this manuscript is concluded in Section 5.

\section{Preliminaries}

2.1. Notations. $\quad \mathbb{R}^{n}$ : the $n$-dimensional real Euclidean space $\mathbb{C}^{n}$ : the $n$-dimensional complex Euclidean space

$\mathbb{R}^{n \times n}$ : the set of $n \times n$ real matrices

$\mathbb{C}^{n \times n}:$ the set of $n \times n$ complex matrices

$H\left(x^{H}\right)$ : the conjugate transpose of a matrix or vector $\top\left(x^{\top}\right)$ : the conjugate transpose of a matrix or vector

$I_{n}$ : the identity matrix with $n$ dimensions

$A^{s} A^{s}=\left(\left(A+A^{\top}\right) / 2\right)$ for $A \in \mathbb{R}^{n \times n}$

$M>0$ for symmetric matrices $M, M$ is a positivedefinite matrix

$M<0$ for symmetric matrices $M, M$ is a negativedefinite matrix

$\lambda_{\max }(M)$ : the largest eigenvalue of $M$

$\lambda_{\min }(M):$ the smallest eigenvalue of $M$

$\|\cdot\|$ : Euclidean norm in complex field 
$i=\sqrt{-1}$ denotes the imaginary unit

$|x|$ : the modulus of a complex number $x$

$\mathfrak{R}(x)$ : its real part

$\mathfrak{I}(x)$ : its imaginary part

$\mathscr{C}\left([-\tau, 0], \mathbb{C}^{n}\right)$ : the set of the continuous vector value function in the complex number fields.

2.2. Model Description. We consider a drive system network consisting of $N$ agents. In this network, each agent is an $n$ dimensional identical nonlinearly dynamics unit, and the drive network can be described as

$$
\begin{aligned}
\dot{x}_{i}(t)= & f\left(t, x_{i}(t), x_{i}\left(t-\tau_{1}(t)\right)\right)+\sum_{k=1}^{N} a_{i k}^{D} \Gamma x_{k}(t) \\
& +\sum_{k=1}^{N} b_{i k}^{D} \Gamma x_{k}\left(t-\tau_{2}(t)\right), \quad i=1,2,3, \ldots, N,
\end{aligned}
$$

where $x_{i}(t)=\left[x_{i 1}(t), x_{i 2}(t), \ldots, x_{i n}(t)\right]^{\top} \in \mathbb{C}^{n}$ is the state vector of the $i$ th agent of the network, $f\left(t, x_{i}(t), x_{i}\left(t-\tau_{1}\right.\right.$ $(t)))=\left[f_{1}\left(t, x_{i}(t), x_{i}\left(t-\tau_{1}(t)\right)\right), f_{2}\left(t, x_{i}(t), x_{i}\left(t-\tau_{1}(t)\right)\right)\right.$, $\left.\ldots, f_{n}\left(t, x_{i}(t), x_{i}\left(t-\tau_{1}(t)\right)\right)\right]^{\top} \in \mathbb{C}^{n}$ is a continuous complex vector-valued function, $\Gamma=\operatorname{diag}\left(\varrho_{1}, \varrho_{2}, \ldots, \varrho_{n}\right) \in$ $\mathbb{R}^{n \times n}$ is an inner coupling matrix satisfying $\varrho_{k}>0$ for $k=1,2, \ldots, n . \quad A^{D}=\left[a_{i k}^{D}\right] \in \mathbb{R}^{n \times n}$ is the outer coupling matrix of the network at time $t$ satisfying $a_{i k}^{D} \geq 0$ for $i \neq k$, $a_{i i}^{D}=-\sum_{k=i, k \neq i}^{N} a_{i k}^{D}, B^{D}=\left[b_{i k}^{D}\right] \in \mathbb{R}^{n \times n}$ is the other one with the time-varying delay $\tau_{2}(t)$ satisfying $b_{i k}^{D} \geq 0$ for $i \neq k$ and $b_{i i}^{D}=-\sum_{k=i, k \neq i}^{N} b_{i k}^{D}$, and $\tau_{1}(t)$ and $\tau_{2}(t)$ are the inner and coupling time-varying delay, respectively, which satisfy $\tau_{1} \geq \tau_{1}(t) \geq 0$ and $\tau_{2} \geq \tau_{2}(t) \geq 0$, and let $\tau=\max \left(\tau_{1}, \tau_{2}\right)$.

The response complex dynamical network with controllers is

$$
\begin{aligned}
\dot{y}_{i}(t)= & f\left(t, y_{i}(t), y_{i}\left(t-\tau_{1}(t)\right)\right)+\sum_{s=1}^{N} a_{i s}^{\mathrm{R}} \Gamma y_{s}(t) \\
& +\sum_{s=1}^{N} b_{i s}^{\mathrm{R}} \Gamma y_{s}\left(t-\tau_{2}(t)\right)+u_{i}(t), \quad i=1,2, \ldots, N,
\end{aligned}
$$

where $y_{i}(t)=\left[y_{i 1}(t), y_{i 2}(t), \ldots, y_{i n}(t)\right]^{\top} \in \mathbb{C}^{n}$ is the state vector of the $j$ th agent of the response network and $f\left(t, y_{i}(t), y_{i}\left(t-\tau_{1}(t)\right)\right)=\left[f_{1}\left(t, y_{i}(t), \quad x_{i}\left(t-\tau_{1}(t)\right)\right), f_{2}\right.$ $\left.\left(t, y_{i}(t), y_{i}\left(t-\tau_{1}(t)\right)\right), \ldots, f_{n}\left(t, y_{i}(t), y_{i}\left(t-\tau_{1}(t)\right)\right)\right]^{\top} \in$ $\mathbb{C}^{n}$ is a continuous vector-valued function. Here, $A^{R}=\left[a_{i k}^{R}\right] \in \mathbb{R}^{n \times n}$ is the outer coupling matrix at time $t$ satisfying $a_{i k}^{R} \geq 0$ for $i \neq k, a_{i i}^{D}=-\sum_{k=i, k \neq i}^{N} a_{i k}^{D}, B^{R}=\left[b_{i k}^{R}\right] \epsilon$ $\mathbb{R}^{n \times n}$ is the outer coupling matrices at time $t-\tau_{2}(t)$ satisfying $b_{i k}^{D} \geq 0$ for $i \neq k$ and $b_{i i}^{D}=-\sum_{k=i, k \neq i}^{N} b_{i k}^{D}$, and $u_{i}(t)$ is the control input.

The initial conditions are

$$
\begin{array}{ll}
x_{i}(s)=\varepsilon_{i}(s), & -\check{\tau}_{1} \leq s \leq 0, i=1,2, \ldots, N, \\
y_{i}(s)=v_{i}(s), & -\check{\tau}_{1} \leq s \leq 0, i=1,2, \ldots, N,
\end{array}
$$

where $\check{\tau}_{1}=\max \left\{\tau_{1}(t), \tau_{2}(t)\right\}, \varepsilon_{i}, v_{i} \in C_{\mathscr{F}_{0}}^{b}\left(\left[-\check{\tau}_{1}, 0\right], \mathbb{C}^{n}\right)$ with the norm $\left\|\varepsilon_{i}\right\|^{2}=\sup _{-\check{\tau}_{1} \leq s \leq 0} \varepsilon_{i}(s)^{H} \varepsilon_{i}(s)$.

Remark 1. The coupling matrices between the driven system and the response system are different. The difficulty is how to design controller $u_{i}(t)$ to achieve the outer synchronization of the driver-response networks and to prove it right.

The aperiodically intermittent pinning control is a very good strategy; the part nodes of the response network are controlled by receiving information of the part nodes of the drive network in the aperiodically intermittent way. To achieve the outer-synchronization objective, the controller is designed in system (2) as follows:

$$
u_{i}(t)= \begin{cases}-d_{i} \Gamma\left(x_{i}(t)-y_{i}(t)\right), & i=1,2, \ldots, l, t \in\left(t_{k}, \omega_{k}\right], \\ 0, & i=l+1, l+2, \ldots, N, t \in\left(t_{k}, \omega_{k}\right], \\ 0, & i=1,2, \ldots, N, t \in\left(\omega_{k}, t_{k+1}\right],\end{cases}
$$

where $d_{i}>0$ for $i=1,2, \ldots, l(l<N)$ are the control gain and denote $D=\operatorname{diag}\left\{d_{1}, d_{2}, \ldots, d_{l}, 0, \ldots, 0\right\} \in \mathbb{R}^{N \times N}$. For any time span, $\left(t_{k}, t_{k+1}\right], t_{0}=0, i=0,1,2, \ldots,\left(t_{k}, \omega_{k}\right]$ is the work time (control time) and $\omega_{k}-t_{k}$ is called the $i$ th control width (control duration), where $t_{k}$ and $\omega_{k}$ denote the start time and the end time of $i$ th control, respectively, while $\left(\omega_{k}, t_{k+1}\right]$ is the rest time and $t_{k+1}-\omega_{k}$ is called the $i$ th rest width.

We define $e_{i}(t)=x_{i}(t)-y_{i}(t)$, and one can obtain the error systems as follows:

$$
\begin{aligned}
\dot{e}_{i}(t)= & f\left(t, x_{i}(t), x_{i}\left(t-\tau_{1}(t)\right)\right)-f\left(t, y_{i}(t), y_{i}\left(t-\tau_{1}(t)\right)\right) \\
& +\sum_{k=1}^{N} a_{i k}^{D} \Gamma x_{k}(t)-\sum_{s=1}^{N} a_{i s}^{R} \Gamma y_{s}(t) \\
& +\sum_{k=1}^{N} b_{i k}^{D} \Gamma x_{k}\left(t-\tau_{2}(t)\right)-\sum_{s=1}^{N} b_{i s}^{R} y_{s}\left(t-\tau_{2}(t)\right)+u_{i}(t) .
\end{aligned}
$$

Definition 1. The drive network (1) and the response network (2) are said to be outer exponential synchronized if there exist positive constants $K$ and $\kappa$ such that

$$
\sum_{i=1}^{N}\left\|x_{i}\left(t, t_{0}, \varepsilon_{i}\right)-y_{i}\left(t, t_{0}, v_{i}\right)\right\| \leq K e^{-\kappa t},
$$

for any initial data $\varepsilon_{i}, v_{i} \in \mathscr{C}\left([-\tau, 0] ; \mathbb{C}^{n}\right)$.

Our first basic assumption will be used throughout this paper to deal with the nonlinearity of the systems.

Assumption 1 (See [19]). For some given matrix $\Gamma$, it is assumed there exists a positive-definite diagonal matrix $P=\operatorname{diag}\left\{p_{1}, p_{2}, \ldots, p_{n}\right\}$, a diagonal matrix $\Delta=\operatorname{diag}\left\{\delta_{1}\right.$, $\left.\delta_{2}, \ldots, \delta_{n}\right\}$, and constants $\eta>0, \zeta>0$ such that such that the complex-valued vector function $f(t, \cdot, \cdot)$ satisfies 


$$
\begin{gathered}
(x-y)^{H} P((f(t, x, z)-f(t, y, w))-\Delta \Sigma(x-y)) \\
\leq-\eta(x-y)^{H}(x-y)+\zeta(z-w)^{H}(z-w),
\end{gathered}
$$

for all $x, y, z, w \in \mathbb{C}^{n}$.

The following basic assumption is that the switching is slow in the sense of combined work time and rest time.

Assumption 2. For the aperiodically intermittent control strategy, there exist two positive scalars $0<\theta<\omega<+\infty$ such that, for $i=0,1,2, \ldots$,

$$
\left\{\begin{array}{l}
\inf _{i}\left(\omega_{i}-t_{i}\right)=\theta, \\
\sup _{i}\left(t_{i+1}-t_{i}\right)=\omega .
\end{array}\right.
$$

Remark 2. In the assumption, $\theta$ and $\omega$ characterize the aperiodically intermittent control. The time span of each control width should be no less than $\theta$, while the sum span of control and rest width should be no larger than $\omega$, i.e., the span of rest width should be no larger than $\omega-\theta$.

2.3. Some Lemmas. In the following, we present some lemmas that will be required throughout this paper.

Lemma 1 (See [26]). Let $y(\cdot):\left[t_{0}-\tau,+\infty\right) \longrightarrow[0,+\infty)$ be a continuous function such that

$$
\dot{y}(t)<a y(t)+b \bar{y}(t)
$$

where $b>0, a+b>0, \bar{y}(t)=\sup _{t-\tau \leq s \leq t} y(s)$; then, for $t \geq t_{0}$,

$$
y(t) \leq \bar{y}\left(t_{0}\right) e^{(a+b)\left(t-t_{0}\right)} \text {. }
$$

Lemma 2 (See [26]). Let $y(\cdot):\left[t_{0}-\tau,+\infty\right) \longrightarrow[0,+\infty)$ be a continuous function such that

$$
\dot{y}(t)<-a y(t)+b \bar{y}(t),
$$

where $a, b$ are positive scalars satisfying $a>b$ and $\bar{y}(t)=\sup _{t-\tau \leq s \leq t} y(s)$; then, for $t \geq t_{0}$,

$$
y(t) \leq \bar{y}\left(t_{0}\right) e^{-r\left(t-t_{0}\right)},
$$

where $r$ is the unique positive solution of

$$
a=b e^{r \tau}+r
$$

Lemma 3 (See [26]). If the continuous and nonnegative function $y(t)$ satisfies

$$
\left\{\begin{array}{ll}
\dot{y}(t)<-a y(t)+b \bar{y}(t), & t_{i} \leq t \leq \omega_{i}, \\
\dot{y}(t)<c y(t)+b \bar{y}(t), & \omega_{i} \leq t \leq t_{i+1},
\end{array} \quad i=0,1,2, \ldots,\right.
$$

for $t \in[-\tau, \infty)$, where $\tau>0, t_{i}$ and $\omega_{i}$ are defined by the aperiodically intermittent controller (8), and we suppose that there exists a constant $\psi$, where $\psi=\limsup i \rightarrow \infty$ $\left(t_{i+1}-\omega_{i} / t_{i+1}-t_{i}\right)$. If $a>b \geq 0, \delta=a+c>0, \varrho=\lambda-\delta \psi>0$, then

$$
y(t) \leq \bar{y}(0) e^{-\varrho t}, \quad t \geq 0,
$$

where $\lambda>0$ is the unique positive solution of the equation $\lambda-a+b e^{\lambda \tau}=0$.

Definition 2. Let $A=\left[a_{i j}\right] \in \mathbb{R}^{m \times n}, B=\left[b_{i j}\right] \in \mathbb{R}^{s \times t}$, and we denote the Kronecker product as follows:

$$
A \otimes B=\left[\begin{array}{cccc}
a_{11} B & a_{12} B & \ldots & a_{1 n} B \\
a_{21} B & a_{22} B & \ldots & a_{2 n} B \\
\vdots & \vdots & \ddots & \vdots \\
a_{m 1} B & a_{m 2} B & \ldots & a_{m n} B
\end{array}\right] \in \mathbb{R}^{m s \times n t} .
$$

\section{Main Results}

Here, via using aperiodically intermittent pinning control, we obtain the synchronization conditions for these two complex dynamic networks which have different complex structures and time-varying delays.

Theorem 1. We suppose Assumptions 1 and 2 hold if there exist a positive-definite matrix $P$ and positive constants $\alpha$ and $\beta$ such that

$$
\mu=\lambda(\theta-\tau)-\nu(\omega-\theta)>0
$$

where $v=a_{2}+2 b$, and $\lambda>0$ is the unique positive solution of the equation

$$
\begin{aligned}
\lambda-a_{1}+b e^{\lambda \tau} & =0, \\
a_{1} & =\lambda_{\min }\left\{(\eta-\alpha-\beta) I_{N n}-I_{N} \otimes(P \Delta \Gamma)-\left(A^{R}+A^{D}-D\right)^{s} \otimes(P \Gamma)\right\}, \\
a_{2} & =\lambda_{\max }\left\{(-\eta+\alpha+\beta) I_{N n}+I_{N} \otimes(P \Delta \Gamma)+\left(A^{R}+A^{D}\right)^{s} \otimes(P \Gamma)\right\}, \\
b & =\zeta+\lambda_{\max }\left\{\left(\frac{1}{\beta}\left(B^{R}\right)^{\top}\left(B^{R}\right)+\frac{1}{\alpha}\left(B^{D}\right)^{\top}\left(B^{D}\right)\right) \otimes(P \Gamma)^{\top}(P \Gamma)\right\} .
\end{aligned}
$$


Then, drive network (1) and response network (2) are outer exponential synchronized via aperiodical intermittent pinning controller (4).

Proof. Basing on the properties of the error systems, one can define a Lyapunov function

$$
V(e(t))=\frac{1}{2} \sum_{i=1}^{N} e_{i}(t)^{H} P e_{i}(t),
$$

when $t \in\left(t_{k}, \omega_{k}\right]$, and one can obtain that

$$
\begin{aligned}
\dot{V}(e(t))= & \sum_{i=1}^{N} e_{i}(t)^{H}\left\{f\left(t, x_{i}(t), x_{i}\left(t-\tau_{1}(t)\right)\right)\right. \\
& -f\left(t, y_{i}(t), y_{i}\left(t-\tau_{1}(t)\right)\right) \\
& +\sum_{k=1}^{N} a_{i k}^{D} \Gamma x_{k}(t)-\sum_{s=1}^{N} a_{i s}^{R} \Gamma y_{s}(t) \\
& +\sum_{k=1}^{N} b_{i k}^{D} \Gamma x_{k}\left(t-\tau_{2}(t)\right) \\
& \left.-\sum_{s=1}^{N} b_{i s}^{R} y_{s}\left(t-\tau_{2}(t)\right)+u_{i}(t)\right\} .
\end{aligned}
$$

For simplicity, we denote $e(t)=\left[e_{1}^{H}(t), e_{2}^{H}(t)\right.$, $\left.\ldots, e_{N}^{H}(t)\right]^{H} \in \mathbb{C}^{n N}$.

According to Assumption 1, one can obtain that

$$
\begin{gathered}
\sum_{i=1}^{N} e_{i}(t)^{H} P\left\{f\left(t, x_{i}(t), x_{i}\left(t-\tau_{1}(t)\right)\right)\right. \\
\left.-f\left(t, y_{i}(t), y_{i}\left(t-\tau_{1}(t)\right)\right)\right\}
\end{gathered}
$$

$$
\begin{aligned}
\leq & -\eta e^{H}(t) e(t)+\zeta e^{H}\left(t-\tau_{1}(t)\right) e\left(t-\tau_{1}(t)\right) \\
& +e^{H}(t) I_{N} \otimes(P \Delta \Gamma) e(t) .
\end{aligned}
$$

From the definitions of $A^{D}\left(\sum_{k=i}^{N} a_{i k}^{D}=0\right)$, the following equation holds:

$$
\begin{aligned}
\sum_{i=1}^{N} e_{i}^{H}(t) P \sum_{k=1}^{N} a_{i k}^{D} \Gamma x_{k}(t) & =\sum_{i=1}^{N} e_{i} P \sum_{k=1}^{N} a_{i k}^{D} \Gamma\left(x_{k}(t)-y_{i}(t)\right) \\
& =e^{H}(t) A^{D} \otimes(P \Gamma) e(t) .
\end{aligned}
$$

Similarly,

$$
\begin{aligned}
\sum_{i=1}^{N} e_{i}^{H}(t) P \sum_{k=1}^{N} b_{i k}^{D} \Gamma x_{k}\left(t-\tau_{2}(t)\right) & =e^{H}(t) B^{D} \otimes(P \Gamma) e\left(t-\tau_{2}(t)\right), \\
\sum_{i=1}^{N} e_{i}^{H}(t) P \sum_{s=1}^{N} a_{i k}^{R} \Gamma x_{s}(t) & =e^{H}(t) A^{R} \otimes(P \Gamma) e(t), \\
\sum_{i=1}^{N} e_{i}^{H}(t) P \sum_{s=1}^{N} b_{i k}^{R} \Gamma x_{s}\left(t-\tau_{2}(t)\right) & =e^{H}(t) B^{R} \otimes(P \Gamma) e\left(t-\tau_{2}(t)\right) .
\end{aligned}
$$

By using $2 x^{H} y \leq c x^{H} x+(1 / c) y^{H} y$ for any $x, y \in \mathbb{C}^{N}$ and $c>0$, one can get

$$
\begin{aligned}
e^{H}(t) B^{D} \otimes(P \Gamma) e\left(t-\tau_{2}(t)\right) \leq & \frac{1}{2} \alpha e^{H}(t) e(t)+\frac{1}{2 \alpha} e\left(t-\tau_{2}(t)\right)\left(B^{D}\right)^{H} B^{D} \otimes(P \Gamma)^{H} \\
& \cdot(P \Gamma) e\left(t-\tau_{2}(t)\right) e^{H}(t) B^{R} \otimes(P \Gamma) e\left(t-\tau_{2}(t)\right) \leq \frac{1}{2} \beta e^{H}(t) e(t) \\
& +\frac{1}{2 \beta} e\left(t-\tau_{2}(t)\right)\left(B^{R}\right)^{H} B^{R} \otimes(P \Gamma)^{H}(P \Gamma) e\left(t-\tau_{2}(t)\right),
\end{aligned}
$$

where $\alpha>0$ and $\beta>0$.

Also, one can get

$$
\sum_{i=1}^{N} e_{i}^{H}(t) P u_{i}(t)=-\sum_{i=1}^{N} e_{i}^{H}(t) P d_{i} \Gamma e_{i 1}(t)=-e^{H}(t) D \otimes(P \Gamma) e(t) .
$$

Substituting inequalities (21)-(25) into equality (20), we get

$$
\begin{aligned}
\dot{V}(t, e(t)) \leq & e^{H}(t)\left\{-\left(\eta-\frac{1}{2} \alpha-\frac{1}{2} \beta\right) I_{N n}+I_{N} \otimes(P \Delta \Gamma)+\left(A^{R}+A^{D}-D\right) \otimes(P \Gamma)\right\} e(t) \\
& +\zeta e^{H}\left(t-\tau_{1}(t)\right) e\left(t-\tau_{1}(t)\right)+e^{H}\left(t-\tau_{2}(t)\right)\left(\left(\frac{1}{2 \beta}\left(B^{R}\right)^{H}\left(B^{R}\right)+\frac{1}{2 \alpha}\left(B^{D}\right)^{H}\left(B^{D}\right)\right) \otimes(P \Gamma)^{H}(P \Gamma)\right) \\
& e\left(t-\tau_{2}(t)\right) .
\end{aligned}
$$


So, we get

$$
\dot{V}(t, e(t)) \leq-a_{1} V(t)+b \sup _{t-\tau \leq s \leq t} V(s) .
$$

$$
\begin{aligned}
& \dot{V}(t, e(t))=\sum_{i=1}^{N} e_{i}(t)^{H} P\left\{\begin{array}{c}
f\left(t, x_{i}(t), x_{i}\left(t-\tau_{1}(t)\right)\right)-f\left(t, y_{i}(t), y_{i}\left(t-\tau_{1}(t)\right)\right) \\
+\sum_{k=1}^{N} a_{i k}^{D \Gamma x_{k}(t)}-\sum_{s=1}^{N} a_{j s}^{R} \Gamma y_{s}(t)+\sum_{k=1}^{N} b_{i k}^{D} \Gamma x_{k}\left(t-\tau_{2}(t)\right) \\
-\sum_{s=1}^{N} b_{j s}^{R} y_{s}\left(t-\tau_{2}(t)\right)
\end{array}\right\} \\
& \leq e^{H}(t)\left\{\left(-\eta+\frac{1}{2} \alpha+\frac{1}{2} \beta\right) I_{N n}+I_{N} \otimes(P \Delta \Gamma)+\left(A^{R}+A^{D}\right) \otimes(P \Gamma)\right\} \\
& e(t)+\zeta e^{H}\left(t-\tau_{1}(t)\right) e\left(t-\tau_{1}(t)\right)+e^{H}\left(t-\tau_{2}(t)\right) \\
& \cdot\left(\left(\frac{1}{2 \beta}\left(B^{R}\right)^{\top}\left(B^{R}\right)+\frac{1}{2 \alpha}\left(B^{D}\right)^{\top}\left(B^{D}\right)\right) \otimes(P \Gamma)^{\top}(P \Gamma)\right) e\left(t-\tau_{2}(t)\right) \text {. }
\end{aligned}
$$

So, we get

$$
\dot{V}(t, e(t)) \leq a_{2} V(t)+b \sup _{t-\tau \leq s \leq t} V(s) .
$$

By Lemma 3 and condition (17), we get

$$
\sum_{i=1}^{N}\left\|e_{i}(t)\right\|^{2} \leq K e^{-\kappa t},
$$

where $K=\exp (\mu+\beta \omega) \sup _{-\tau \leq s \leq 0} V(s)$ and $\kappa=(\mu / \nu)>0$. The proof is completed.
Remark 3. The condition of outer synchronization is given in (17) and (18) and is affected due to the different coupling matrices between the driven system and the response system.

When the coupling is without delay, that is, $\tau_{2}(t)=0$, then we have the corollary as follows.

Corollary 1. We suppose Assumptions 1 and 2 hold, a positive-definite matrix $P$, and positive constants $\alpha$ and $\beta$ such that

$$
\mu=\lambda(\theta-\tau)-\nu(\omega-\theta)>0
$$

where $v=a_{2}+2 b$, and $\lambda>0$ is the unique positive solution of the equation

$$
\begin{aligned}
\lambda-a_{1}+b e^{\lambda \tau} & =0, \\
a_{1} & =\lambda_{\min }\left\{(\eta-\alpha-\beta) I_{N n}-I_{N} \otimes(P \Delta \Gamma)-\left(A^{R}+A^{D}-D\right)^{s} \otimes(P \Gamma)\right\}, \\
a_{2} & =\lambda_{\max }\left\{(-\eta+\alpha+\beta) I_{N n}+I_{N} \otimes(P \Delta \Gamma)+\left(A^{R}+A^{D}\right)^{s} \otimes(P \Gamma)\right\}, \\
b & =\zeta .
\end{aligned}
$$

Then, the solution $e_{i}(t), i=1, \ldots, N$ of system (5) is globally exponentially stabilized with the aperiodical intermittent pinning controller (4).
Remark 4. The corollary gives the sufficient conditions to ensure the drive systems and the response system to achieve synchronization by the aperiodical intermittent pinning 


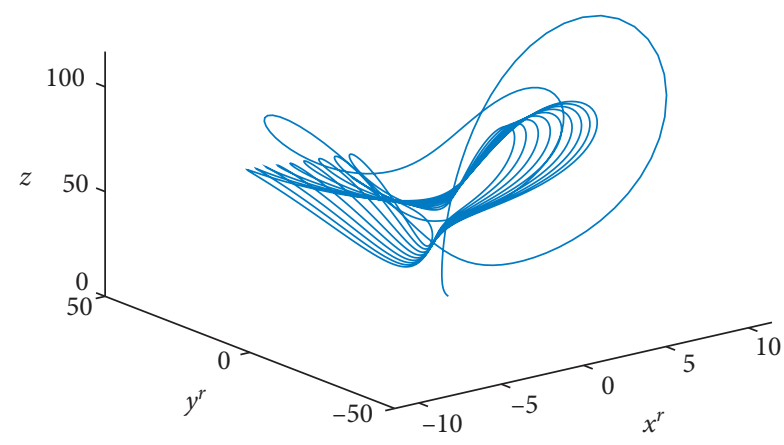

(a)

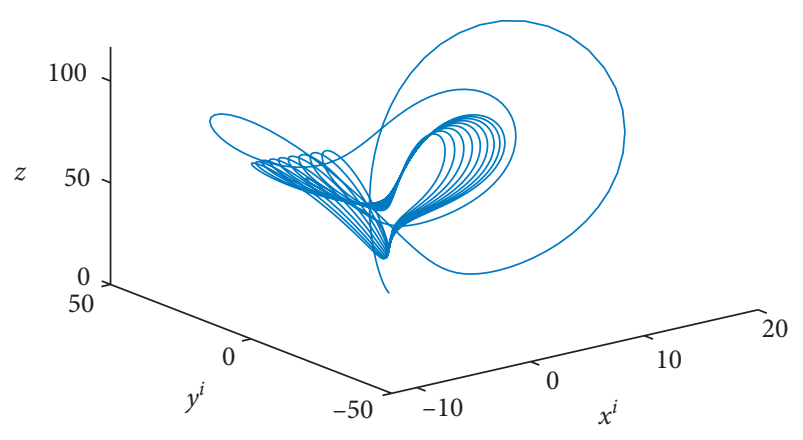

(b)

FIgURE 1: A complex-valued Lorenz system is illustrated. (a) The image part of the states. (b) The real part of the states.

controller, which extends the result of [13], where the states of the drive systems and the response systems are real and the coupling is without the delay.

\section{Numerical Simulation}

In this section, one numerical simulation example is presented to validate the effectiveness of the abovementioned theorem by the complex-value Lorenz system.

$$
\left\{\begin{array}{l}
\dot{x}=-a_{1} x+a_{1} y, \\
\dot{y}=\left(a_{3}-z\right) x-a_{4} y, \\
\dot{z}=-a_{2} z+\frac{1}{2}(\bar{x} y+x \bar{y}) .
\end{array}\right.
$$

It has a chaotic attractor when the parameters $a_{1}=2$, $a_{2}=0.8, a_{3}=60+0.02 i$, and $a_{4}=1-0.06 i$ [17]. Figure 1 shows the state of the complex-valued Lorenz system with the initial value $(1+i, 2+4 i, 3)$. One can get $\mathfrak{J}(z(t))=0$ if $\mathfrak{J}(z(0))=0$.

We consider the driver-response system of 10 agents via coupling protocol (1), (2), and (4) with the topology as shown in Figure 2. Let

$$
\begin{aligned}
\dot{x}_{i}(t)= & f\left(t, x_{i}(t), x_{i}\left(t-\tau_{1}(t)\right)\right)+\sum_{j=1}^{10} a_{i k}^{D} \Gamma x_{k}(t)+\sum_{k=1}^{10} b_{i k}^{D} \Gamma x_{k}\left(t-\tau_{2}(t)\right) \\
& -d_{i} \Gamma \sum_{j=1}^{2}\left(x_{i}(t)-y_{i}(t)\right), \quad i=1,2, \ldots, 10, \\
\dot{y}_{i}(t)= & f\left(t, y_{i}(t), y_{i}\left(t-\tau_{1}(t)\right)\right)+\sum_{s=1}^{10} a_{i s}^{R} \Gamma y_{s}(t)+\sum_{s=1}^{10} b_{i s}^{R} \Gamma y_{s}\left(t-\tau_{2}(t)\right), \quad i=1,2, \ldots, 10 .
\end{aligned}
$$

In this numerical simulation, let $D=\operatorname{diag}$ $\{1,1,1,1,1,0,0,0,0,0\}, \quad \tau_{2}(t)=0.01\left(e^{t} / 1+e^{t}\right), \quad l^{\mathrm{D}}=2$, $\sigma_{i}\left(t, x_{i}(t), x_{i}\left(t-\tau_{1}(t)\right), \quad x_{i}\left(t-\tau_{2}(t)\right)\right)=0.01 \operatorname{diag}\left\{x_{i 1}(t)+\right.$ $x_{i 1}\left(t-\tau_{1}(t)\right)+x_{i 1} \quad\left(t-\tau_{2}(t)\right), x_{i 2}(t)+x_{i 2}\left(t-\tau_{1}(t)\right)+x_{i 2}$ $\left.\left(t-\tau_{2}(t)\right)\right\}$, and $\sigma_{i}\left(t, y_{i}(t), y_{i}\left(t-\tau_{1}(t)\right), y_{i}\left(t-\tau_{2}(t)\right)\right)=$ $0.01 \operatorname{diag}\left\{y_{i 1}(t)+y_{i 1}\left(t-\tau_{1}(t)\right)+y_{i 1}\left(t-\tau_{2}(t)\right), y_{i 2}(t)+y_{i 2}\right.$ $\left.\left(t-\tau_{1}(t)\right)+y_{i 2}\left(t-\tau_{2}(t)\right)\right\}$.

Computations then yield $\tau_{1}=1, \bar{\tau}_{1}=0, \tau_{2}=0.01$, and $\bar{\tau}_{1}=0.0025$. One can design the control strength $d_{i}=100$. By simple computation, one can obtain that $a_{1}=5.8039$, $b=3.260, c=2.4443, a_{2}=1042.3$, and $\gamma=0.0138$ by solving equation (18) in Theorem 1.

The initial values for this simulation are $x_{i j}\left(t_{0}\right)=$ $-2 \times i \times \cos \left(t_{0}\right)-k \times \cos \left(t_{0}\right), \quad y_{j s}\left(t_{0}\right)=-2 \times \quad(i+7) \times$ $\cos \left(t_{0}\right)+s \times \cos \left(t_{0}\right)$, for $i=1, \ldots, 10, j=1, \ldots, 7, k=1,2$, and $t_{0} \in[-1,0]$. The trajectories of system (35) and (34) under the aperiodically intermittent pinning control gains are shown in Figures 3 and 4. It is obvious that the outer synchronization between (35) and (34) can be realized. 


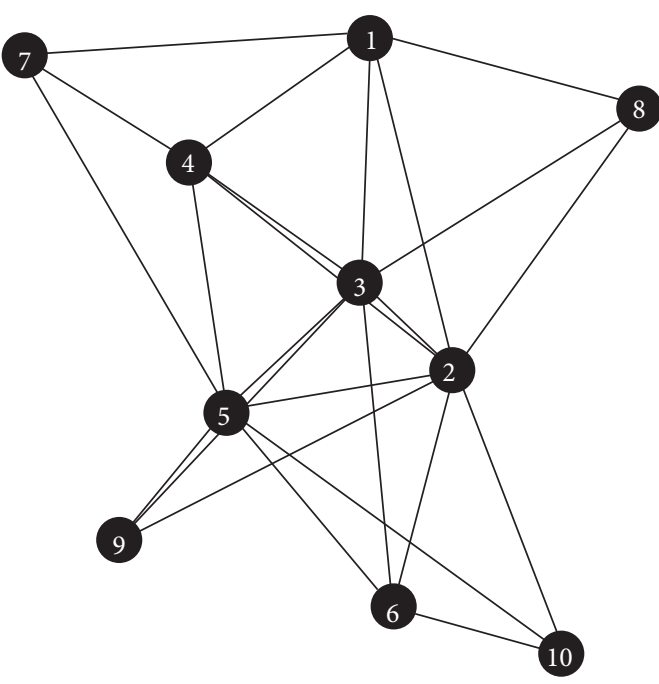

(a)

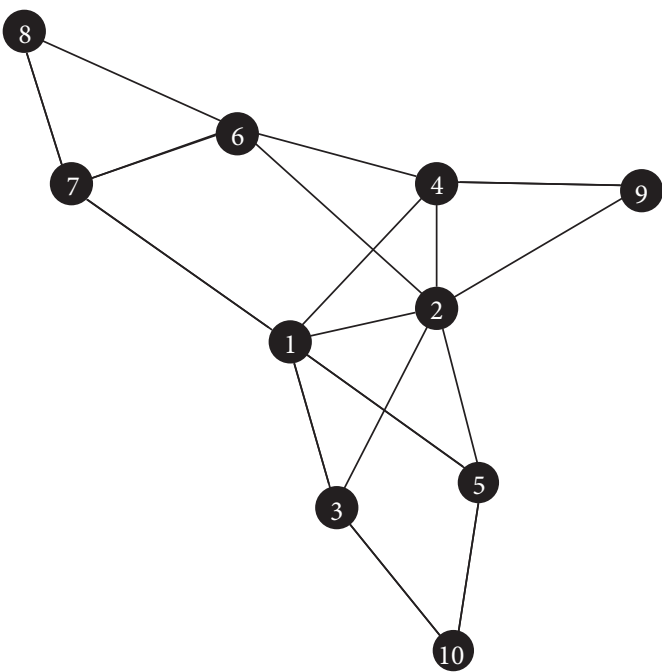

(c)

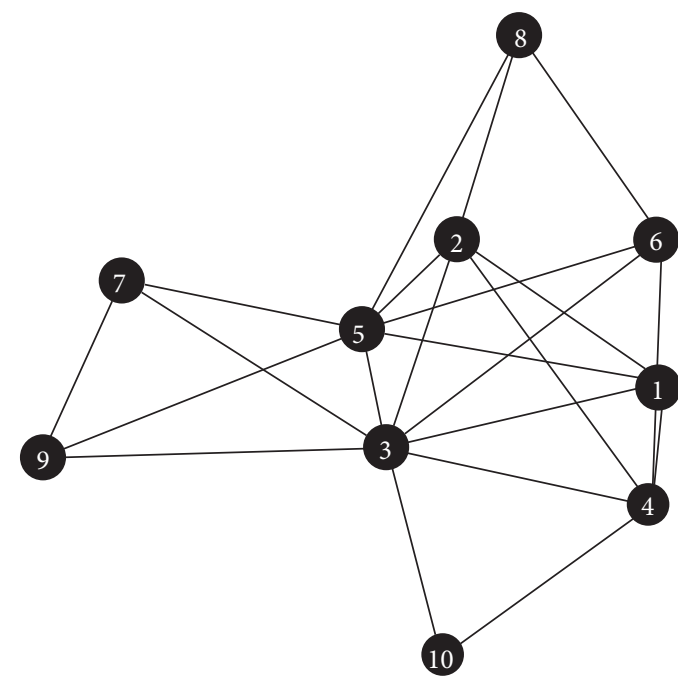

(b)

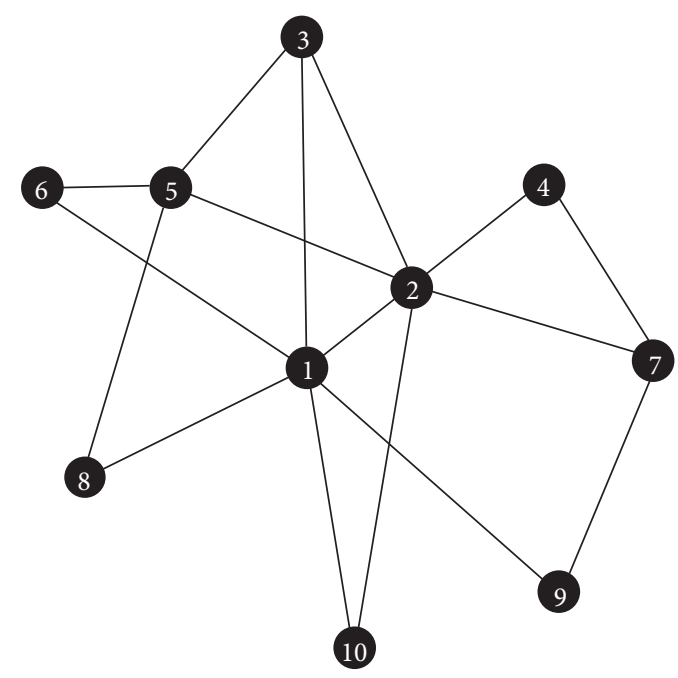

(d)

FiguRe 2: The topology structures of the response (a, b) and drive (c, d) network. (a) $A^{\mathrm{R}}$, (b) $B^{\mathrm{R}}$, (c) $A^{\mathrm{D}}$, (d) $B^{\mathrm{D}}$.

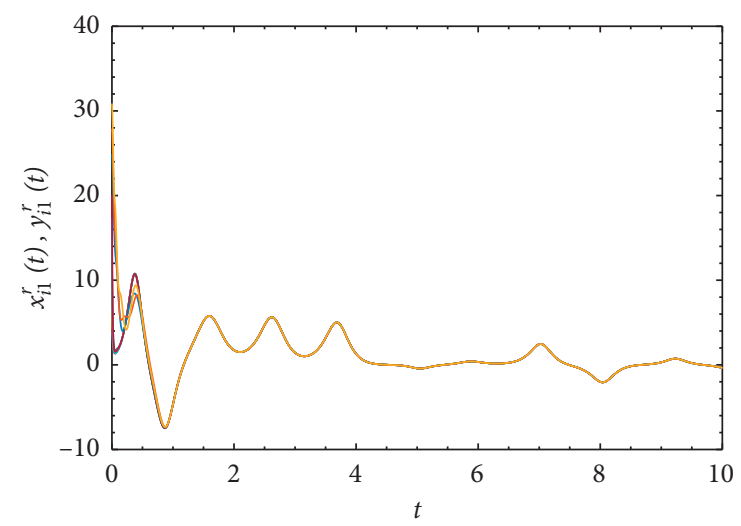

(a)

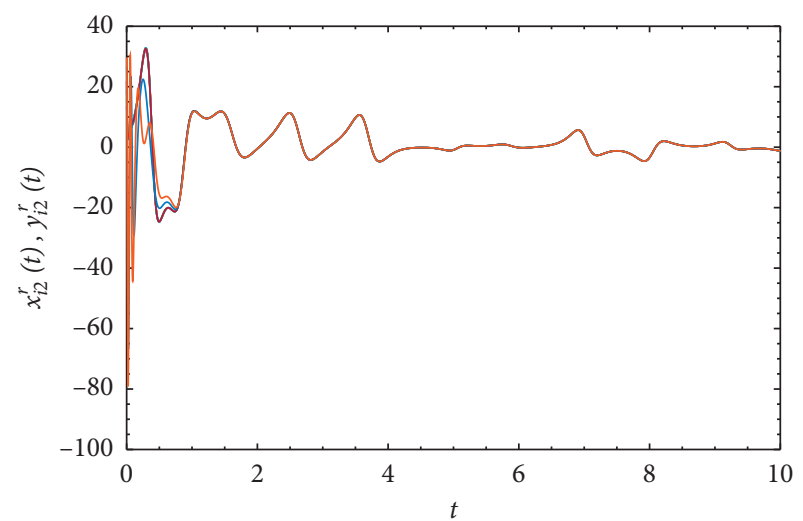

(b)

Figure 3: Continued. 


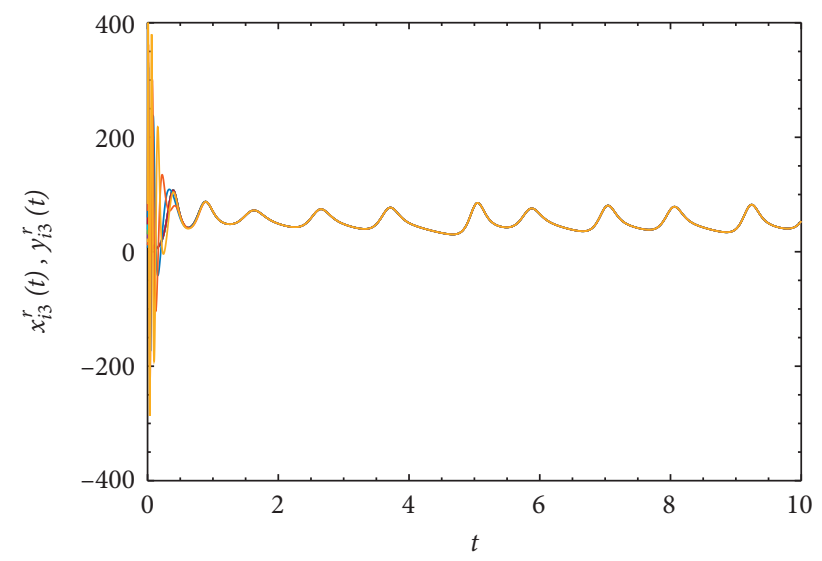

(c)

FIgURE 3: The evolution of $x_{i 1}$ and $y_{i 1}(i=1,2, \ldots, 10)$ in system (34) and (35) via aperiodically intermittent control.

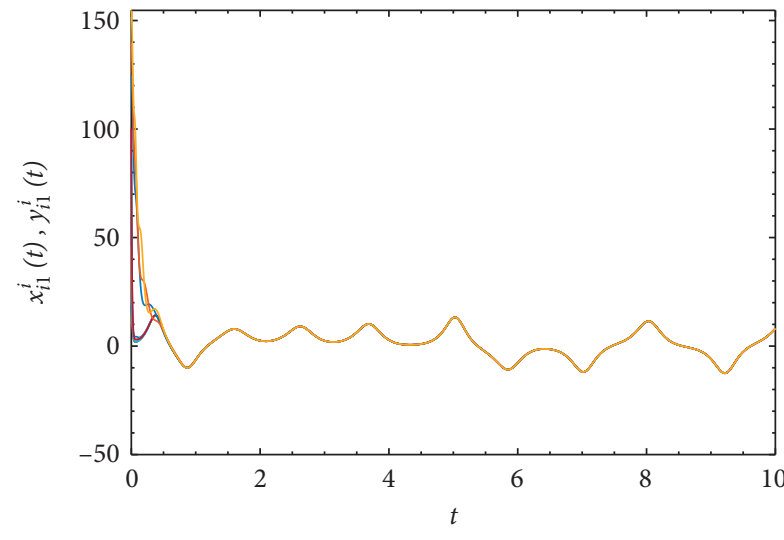

(a)

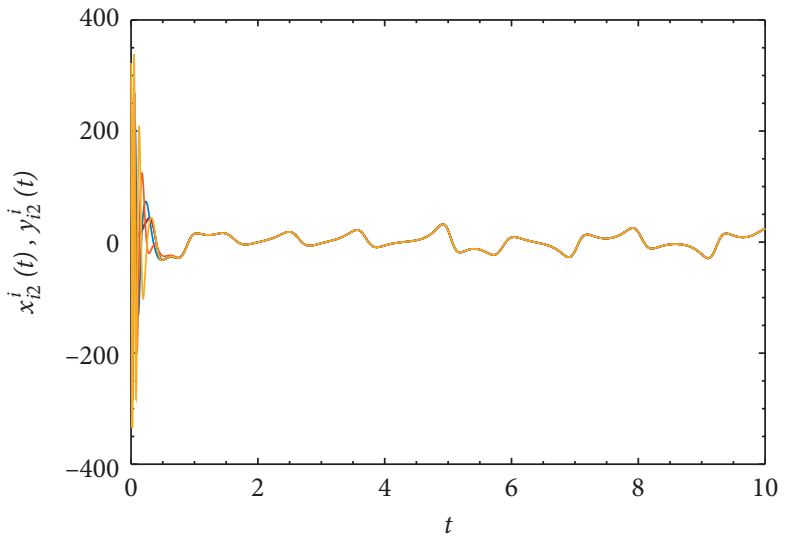

(b)

FIgURE 4: The evolution of $x_{i 2}$ and $j_{i 2}(i=1,2, \ldots, 10)$ in system (34) and (35) via aperiodically intermittent control.

\section{Conclusions}

In this paper, the outer-synchronization problem of two complex-value complex dynamical networks with node delay and time-varying coupling delay is investigated. In particular, we realized global outer synchronization by using an aperiodically intermittent control scheme to a small part of agents. Furthermore, we obtained sufficient criteria for the global exponential outer synchronization. Finally, one numerical example is given to illustrate the theoretical analysis.

In the future, the work will be extended to the case that the drive network and response network are coupled via negatively weighted couplings. The event-trigger intermittent control will be considered.

\section{Data Availability}

The data used to support the findings of this study are available from the corresponding author upon request.

\section{Conflicts of Interest}

The authors declare that there are no conflicts of interest regarding the publication of this paper.

\section{Acknowledgments}

This work was supported by the Shenzhen Science and Technology Program under Grant no. JCYJ20160428092427867 and the Department of Education of Guangdong Province Technological Innovation Program under Grant 2017GKTSCX060.

\section{References}

[1] A. Li, S. P. Cornelius, Y.-Y. Liu, L. Wang, and A.-L. Barabási, "The fundamental advantages of temporal networks," Science, vol. 358, no. 6366, pp. 1042-1046, 2017.

[2] K. Raza, "Network biology: a new way to understand complex disease and future of medicine," CSI Communications, vol. 38, no. 10, 2015. 
[3] S. Fortunato, C. T. Bergstrom, K. Börner et al., "Science of Science," Science, vol. 359, no. 6379, Article ID eaao0185, 2018.

[4] L. Lv, C. Li, L. Chen et al., "Outer synchronization between uncertain networks with adaptive scaling function and different node numbers," Physica A: Statistical Mechanics and its Applications, vol. 506, pp. 909-918, 2018.

[5] J. Wang, J. Feng, C. Xu, M. Z. Q. Chen, Y. Zhao, and J. Feng, "The synchronization of instantaneously coupled harmonic oscillators using sampled data with measurement noise," Automatica, vol. 66, pp. 155-162, 2016.

[6] J. Wang, C. Xu, M. Z. Q. Chen, J. Feng, and G. Chen, "Stochastic feedback coupling synchronization of networked harmonic oscillators," Automatica, vol. 87, pp. 404-411, 2018.

[7] X. Liu, P. Li, and T. Chen, "Cluster synchronization for delayed complex networks via periodically intermittent pinning control," Neurocomputing, vol. 162, pp. 191-200, 2015.

[8] G. Velmurugan and R. Rakkiyappan, "Hybrid projective synchronization of fractional-order memristor-based neural networks with time delays," Nonlinear Dynamics, vol. 11, no. 3, pp. 1-14, 2015.

[9] J. Wang, J. Feng, C. Xu, and Y. Zhao, “Almost sure exponential synchronisation of networked harmonic oscillators via intermittent coupling subject to Markovian jumping," IET Control Theory \& Applications, vol. 12, no. 11, pp. 1658-1664, 2018.

[10] X. Li, X. Yang, and T. Huang, "Persistence of delayed cooperative models: impulsive control method," Applied Mathematics and Computation, vol. 342, pp. 130-146, 2019.

[11] X. Li, J. Shen, and R. Rakkiyappan, "Persistent impulsive effects on stability of functional differential equations with finite or infinite delay," Applied Mathematics and Computation, vol. 329, pp. 14-22, 2018.

[12] A. Ray and A. Roychowdhury, "Outer synchronization of networks with different node dynamics," The European Physical Journal Special Topics, vol. 223, no. 8, pp. 1509-1518, 2014.

[13] J. Lu, C. Ding, J. Lou, and J. Cao, "Outer synchronization of partially coupled dynamical networks via pinning impulsive controllers," Journal of the Franklin Institute, vol. 352, no. 11, pp. 5024-5041, 2015.

[14] L. Lü, C. Li, L. Chen et al., "Outer synchronization between uncertain networks with adaptive scaling function and different node numbers," Physica A: Statistical Mechanics and its Applications, vol. 506, pp. 909-918, 2018.

[15] L. Zhang, Y. Lei, Y. Wang, and H. Chen, "Generalized outer synchronization between non-dissipatively coupled complex networks with different-dimensional nodes," Applied Mathematical Modelling, vol. 55, pp. 248-261, 2017.

[16] C. Zhang, X. Wang, S. Wang, W. Zhou, and Z. Xia, "Finitetime synchronization for a class of fully complex-valued networks with coupling delay," IEEE Access, vol. 6, Article ID 2818192, 2018.

[17] Z. Wu, D. Liu, and Q. Ye, "Pinning impulsive synchronization of complex-variable dynamical network," Communications in Nonlinear Science and Numerical Simulation, vol. 20, no. 1, pp. 273-280, 2015.

[18] J. Sun, W. Deng, G. Cui, and Y. Wang, "Real combination synchronization of three fractional-order complex-variable chaotic systems," Optik, vol. 127, no. 23, pp. 11460-11468, 2016.

[19] Q. Xu, S. Zhuang, S. Liu, and J. Xiao, "Decentralized adaptive coupling synchronization of fractional-order complex- variable dynamical networks," Neurocomputing, vol. 186, pp. 119-126, 2016.

[20] G. Zhou, C. Li, T. Li et al., "Outer synchronization investigation between WS and NW small-world networks with different node numbers," Physica A: Statistical Mechanics and its Applications, vol. 457, pp. 506-513, 2016.

[21] Z. Wu, X. Xu, G. Chen, and X. Fu, "Generalized matrix projective synchronization of general colored networks with different-dimensional node dynamics," Journal of the Franklin Institute, vol. 351, no. 9, pp. 4584-4595, 2014.

[22] L. Zhang, Y. Lei, Y. Wang, and B. Chen, "Stabilization of timevarying and disturbed complex dynamical networks with different-dimensional nodes and uncertain nonlinearities," Asian Journal of Control, vol. 19, no. 6, pp. 2143-2154, 2017.

[23] L. Zhang, Y. Lei, Y. Wang, and H. Chen, "Generalized outer synchronization between non-dissipatively coupled complex networks with different-dimensional nodes," Applied Mathematical Modelling, vol. 55, pp. 248-261, 2018.

[24] S. Cai, X. Lei, and Z. Liu, "Outer synchronization between two hybrid-coupled delayed dynamical networks via aperiodically adaptive intermittent pinning control," Complexity, vol. 21, no. S2, pp. 593-605, 2016.

[25] X. Lei, S. Cai, S. Jiang, and Z. Liu, "Adaptive outer synchronization between two complex delayed dynamical networks via aperiodically intermittent pinning control," Neurocomputing, vol. 222, pp. 26-35, 2017.

[26] X. Liu and T. Chen, "Synchronization of nonlinear coupled networks via aperiodically intermittent pinning control," IEEE Transactions on Neural Networks and Learning Systems, vol. 26, no. 1, pp. 113-126, 2015. 